\title{
Inflow Mechanism for Hydrodynamic Entropy
}

\author{
Felix M. Haehl, ${ }^{1 *}$ R. Loganayagam, ${ }^{2, \dagger}$ and Mukund Rangamani ${ }^{3, *}$ \\ ${ }^{1}$ Department of Physics and Astronomy, University of British Columbia, \\ 6224 Agricultural Road, Vancouver, British Columbia V6T 1Z1, Canada \\ ${ }^{2}$ International Centre for Theoretical Sciences (ICTS-TIFR), Shivakote, Hesaraghatta Hobli, Bengaluru 560089, India \\ ${ }^{3}$ Center for Quantum Mathematics and Physics (QMAP) and Department of Physics, University of California,
}

Davis, California 95616, USA

(Received 6 April 2018; revised manuscript received 29 June 2018; published 3 August 2018)

\begin{abstract}
We argue that entropy production in hydrodynamics can be understood via a superspace inflow mechanism. Our arguments are based on a recently developed formalism for constructing effective actions for Schwinger-Keldysh observables in quantum field theories. The formalism explicitly incorporates microscopic unitarity and the Kubo-Martin-Schwinger thermal periodicity conditions, by recasting them in terms of topological Becchi-Rouet-Stora-Tyutin symmetries of the effective action.
\end{abstract}

DOI: 10.1103/PhysRevLett.121.051602

Introduction.-Nonlinear hydrodynamics, the longwavelength, low-energy theory of near-equilibrium systems, is traditionally characterized by the following set of axioms [1,2]: (H1) The low-energy variables are the fluid velocity $u^{\mu}\left(u^{\mu} u_{\mu}=-1\right)$ and intensive thermodynamic parameters such as the temperature $T$. We combine these into the thermal vector $\boldsymbol{\beta}^{\mu}=u^{\mu} / T$. (H2) The currents are functionals of fluid variables and background sources: $T^{\mu \nu}\left[u^{\mu}, T, g_{\mu \nu}\right]$. They admit a gradient expansion with each independent tensor structure defining a potential transport coefficient. (H3) Dynamics is contained in conservation laws: $\nabla_{\mu} T^{\mu \nu}=0$ for energy-momentum. (H4) Admissible constitutive relations require the existence of an entropy current $J_{S}^{\mu}$, which satisfies a local form of the second law of thermodynamics, $\nabla_{\mu} J_{S}^{\mu}=\Delta \geq 0$, for every physical fluid configuration.

While it is easy to intuit that the low-energy theory is characterized by conservation laws, as only the corresponding modes survive to dominate the late-time, long-distance behavior, the entropy current is more mysterious. For one, it emerges in the infrared without being manifest in the ultraviolet and appears in gravitational systems to be associated with black hole horizons. This stark dichotomy can be seen in the context of the fluid-gravity correspondence [3], where the conserved currents live on the boundary of the anti-de Sitter spacetime, while the entropy current is obtained from the interior by pulling back the area form of the horizon [4].

Published by the American Physical Society under the terms of the Creative Commons Attribution 4.0 International license. Further distribution of this work must maintain attribution to the author(s) and the published article's title, journal citation, and DOI. Funded by SCOAP.
Given this status quo, one can ask whether there is a more intuitive way to understand the origin of the hydrodynamic entropy current $J_{S}^{\mu}$ and its associated entropy production $\Delta$ ? We argue here for the following statements [5]. (i) For systems in local equilibrium, the macroscopic free energy current, the Legendre transform of entropy current, $N^{\mu}=J_{S}^{\mu}+\boldsymbol{\beta}_{\nu} T^{\mu \nu}$, is a Noether current for thermal diffeomorphisms along $\boldsymbol{\beta}^{\mu}$. (ii) The hydrodynamic effective theory is one where these thermal diffeomorphisms are gauged as a topological or Becchi-Rouet-Stora-Tyutin (BRST) gauge symmetry, leading to the idea of thermal equivariance. This symmetry can be made manifest by formulating the theory in superspace - an extension of ordinary spacetime by two anticommuting directions. (iii) While the net entropy being conjugate to the gauged thermal diffeomorphisms is conserved, entropy in physical space is produced by virtue of it being sourced in the superspace directions; i.e., there is an inflow of entropy from superspace. This inflow is nonzero when appropriate superspace components of the field strength are turned on. The mechanism for entropy production is thus analogous to the picture for ' $t$ Hooft anomalies in field theories. The inflow mechanism [7] implies that a theory with a 't Hooft anomaly can be coupled to a higherdimensional topological field theory so as to leave the combined system anomaly-free. In the presence of background field strengths, the physical theory acquires its anomaly through an inflow from the topological sector. In the present context, the symmetry under question is thermal diffeomorphisms, which involve taking fields around a fiducial Euclidean circle, and the inflow is from the topological sector of this theory, which has been recast into a superspace description. An appropriate superspace field strength drives this "entropy anomaly" in the physical space.

Hydrodynamic effective field theories.-Any interacting quantum field theory (QFT) will attain thermal equilibrium 
in a stationary spacetime with a timelike Killing field $K^{\mu}$ $[8,9]$. Equivalently, equilibrium questions in a thermal QFT can be answered by a statistical field theory formulated on a Riemannian manifold $\mathcal{M}_{\boldsymbol{\beta}}$ with the Euclidean thermal circle fibered over a spatial base. The generating functional for current correlators is the equilibrium partition function on this nontrivial background. Such configurations are hydrostatic: The fluid velocity points along the Killing field, $\boldsymbol{\beta}^{\mu}=K^{\mu}$, and the local temperature is given by its norm. The free energy current for the fluid is precisely given by the Noether current for diffeomorphisms along the thermal circle [10] — this may be intuitively understood by recalling the fact that stationary black hole entropy is also obtained as a Noether charge [11].

Off equilibrium, $\boldsymbol{\beta}^{\mu}$ ceases to be a Killing vector, and one has to confront challenges of defining entropy, free energy, etc. However, Bhattacharyya's theorem [12] states that a local free energy current exists in near-equilibrium hydrodynamic settings satisfying a local form of the second law as long as the hydrostatic limit $\left(\boldsymbol{\beta}^{\mu} \rightarrow K^{\mu}\right)$ is consistent with the equilibrium partition function. Remarkably, the second law of thermodynamics requires in addition only that leading-order dissipative terms are sign-definite (e.g., viscosities and conductivities are non-negative).

More generally, hydrodynamic transport can be classified into eight distinct classes by examining the off-shell entropy production statement embodied in the adiabaticity equation $[10,13]$ :

$$
\nabla_{\mu} N^{\mu}-\frac{1}{2} T^{\mu \nu} £_{\beta} g_{\mu \nu}=\Delta \geq 0 .
$$

Dissipative solutions to this equation comprise a single class; the remaining seven are adiabatic or nondissipative (i.e., $\Delta=0$ ). Dissipative constitutive relations are characterized by a general symmetric 4-tensor built from the fluid data:

$$
T_{\text {diss }}^{\mu \nu}=\frac{1}{2} \boldsymbol{\eta}^{(\mu \nu)(\rho \sigma)} \mathfrak{f}_{\beta} g_{\rho \sigma}, \quad \boldsymbol{\eta}^{(\mu \nu)(\rho \sigma)}=\boldsymbol{\eta}^{(\rho \sigma)(\mu \nu),}
$$

which provides a non-negative definite inner product on the space of symmetric two tensors:

$$
\Delta=\frac{1}{4} \boldsymbol{\eta}^{(\mu \nu)(\rho \sigma)} \mathfrak{£}_{\beta} g_{\mu \nu} £_{\beta} g_{\rho \sigma} \geq 0 .
$$

The curiosity of this classification is that the remaining seven classes of transport do not lead to entropy production-fluid transport with $\Delta=0$ is surprisingly rich. Given that the adiabaticity equation is off shell (i.e., fluid conservation equations are not imposed), one should ask for a general principle that explains their presence beyond hydrostatics.

Hydrodynamic transport is captured by response functions, which are causal Green's functions, obtained by starting with a system in global equilibrium, disturbing it by turning on a sequence of time-dependent background sources, and finally making a measurement of the effect. In the Schwinger-Keldysh (SK) formalism phrased in the Keldysh average-difference basis [14,15], response functions are correlators of a sequence of difference operators (the disturbances) followed by an average operator (the measurement) in the future. This is the first nonvanishing correlation function, since pure difference operator correlators are constrained to vanish by unitarity [16]. Furthermore, the response functions are related by the Kubo-Martin-Schwinger (KMS) conditions to correlators with multiple average operators $[17,18]$; the latter capture fluctuations, a subset of which are hydrodynamic [19].

How should one implement these constraints at the level of an effective action for coarse-grained degrees of freedom (d.o.f.)? It has been argued by several groups that a topological BRST symmetry is the essential tool for encoding the SK Ward identities and that the KMS condition can be imposed as a further symmetry principle [21-32]. In the remainder of this section, we present a proposal for implementing all constraints arising from unitarity and the KMS condition in terms of a supersymmetric structure: We shall list the essential constraints on the theory and how to implement them as symmetries. This Letter focuses on exploring the physical consequences of such a proposal, and we refer to Ref. [35] for a more detailed description of its origin.

A. Unitarity. The macroscopic theory has two sets of d.o.f: the average or classical fields and the difference or quantum or stochastic fluctuation fields. Consistent couplings are dictated by the ghost d.o.f. which form part of a BRST multiplet $[25,36]$. The BRST symmetries constrain the low-energy dynamics by enforcing the correct influence functionals [37], which arise from integrating out the high-energy modes. This information can be succinctly encoded by working in a superspace that is locally $\mathbb{R}^{d-1,1 \mid 2}$ with coordinates $z^{I}=\left\{\sigma^{a}, \theta, \bar{\theta}\right\}$. Lifting the field content to superfields and writing an effective action in superspace will make the supersymmetry manifest.

B. KMS condition. While the superspace description encodes SK unitarity constraints, imposing the KMS conditions requires additional structure which may be phrased in the framework of equivariant cohomology $[25,26]$. We imagine out-of-equilibrium systems in real time as possessing a local thermal circle fibered over the Lorentzian base space. This fibration is captured by a background timelike vector superfield $\stackrel{\boldsymbol{\beta}}{\boldsymbol{\beta}}^{I}(z)$, which is the superspace lift of the thermal vector [38]. Working in the high-temperature limit, one may then argue that the BRST symmetries should be covariantized with respect to a thermal $U(1)_{\mathrm{T}}$ gauge symmetry, which implements invariance under thermal diffeomorphisms [39]. This is simply a local version of well-known constraints on systems in global equilibrium. On scalar superfields $\AA^{\prime}=\Lambda^{\prime}+\theta \Lambda_{\bar{\psi}}^{\prime}+\bar{\theta} \Lambda_{\psi}^{\prime}+\bar{\theta} \theta \tilde{\Lambda}^{\prime}$, the 
$U(1)_{\mathrm{T}}$ symmetry transformation acts as $\AA^{\prime} \mapsto \AA^{\prime}+\left(\AA_{\Lambda}, \AA^{\prime}\right)_{\beta}$ with

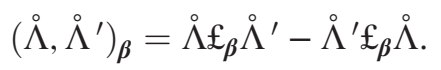

An effective action invariant under this symmetry involves $U(1)_{\mathrm{T}}$ gauge potentials, covariant derivatives, and field strengths which are given in superspace by [26]

$$
\begin{aligned}
& \stackrel{\circ}{\mathcal{A}}_{I}(z) d z^{I}=\stackrel{\mathcal{A}}{a}_{a}(z) d \sigma^{a}+\stackrel{\mathcal{A}}{\theta}_{(}(z) d \theta+\stackrel{\mathcal{A}}{\bar{\theta}}_{(z)}(\bar{\theta}, \\
& \stackrel{\circ}{\mathcal{D}}_{I}=\partial_{I}+\left(\stackrel{\circ}{\mathcal{A}}_{I}, \cdot\right)_{\beta}, \\
& \stackrel{\circ}{\mathcal{F}}_{I J} \equiv\left(1-\frac{1}{2} \delta_{I J}\right)\left[\partial_{I} \stackrel{\AA}{\mathcal{A}}_{J}-(-)^{I J} \partial_{J} \stackrel{\circ}{\mathcal{A}}_{I}+\left(\stackrel{\mathcal{A}}{I}_{I}, \stackrel{\mathcal{A}}{J}_{J}\right)_{\beta}\right] .
\end{aligned}
$$

The hydrodynamic effective field theory in the hightemperature limit is thus constrained by a BRST superalgebra [24] with Grassmann-odd supercharges $\mathcal{Q}, \overline{\mathcal{Q}}$ satisfying

$$
\mathcal{Q}^{2}=-\stackrel{\circ}{\mathcal{F}}_{\bar{\theta} \bar{\theta}}\left|\mathfrak{f}_{\boldsymbol{\beta}}, \quad \overline{\mathcal{Q}}^{2}=-\stackrel{\circ}{\mathcal{F}}_{\theta \theta}\right| \mathfrak{f}_{\beta}, \quad\{\mathcal{Q}, \overline{\mathcal{Q}}\}=-\stackrel{\circ}{\mathcal{F}}_{\theta \bar{\theta}} \mid \mathfrak{f}_{\beta},
$$

where $\mid$ denotes restriction to $\theta=\bar{\theta}=0$. A closely related algebra has appeared in the statistical mechanics literature in the context of stochastic Langevin dynamics [6]. This is also the high-temperature version of the BRST structure identified in Ref. [23] that can be recovered from (6) by setting $\stackrel{\circ}{\mathcal{F}}_{\bar{\theta} \bar{\theta}}=\stackrel{\circ}{\mathcal{F}}_{\theta \theta}=0$ and $\stackrel{\circ}{\mathcal{F}}_{\theta \bar{\theta}}=-i$.

C. Diffeomorphism invariance. A hydrodynamic effective action ought to capture the response functions and the attendant hydrodynamic fluctuations. To this end, note that hydrodynamic variables capture the universal low-energy dynamics in near-equilibrium states for any interacting quantum system. The pion fields of hydrodynamics are maps $X^{\mu}\left(\sigma^{a}\right)$ from a reference world volume to the physical spacetime, such that the push forward of a reference thermal vector gives the physical thermal vector (this is a modern version of the "Lagrangian" description of a fluid). They may be viewed as breaking the individual diffeomorphisms of the SK construction to the diagonal [40]. Incorporation of the BRST symmetries can be done easily by lifting $X^{\mu}$ to superspace:

$$
\stackrel{\circ}{X}^{\mu}=X^{\mu}+\theta X_{\bar{\psi}}^{\mu}+\bar{\theta} X_{\psi}^{\mu}+\bar{\theta} \theta\left(\tilde{X}^{\mu}-\Gamma_{\rho \sigma}^{\mu} X_{\bar{\psi}}^{\rho} X_{\psi}^{\sigma}\right) .
$$

The bottom component of this superfield, $X^{\mu}$, is the classical hydrodynamic field, and the physical thermal vector is the push forward of the background thermal vector, $\boldsymbol{\beta}^{\mu}=\stackrel{\circ}{\boldsymbol{\beta}}^{I} \stackrel{\circ}{\mathcal{D}}_{I} \stackrel{\circ}{X}^{\mu} \mid$. The top component is the fluctuation field $\tilde{X}^{\mu}$, while $X_{\psi}^{\mu}$ and $X_{\bar{\psi}}^{\mu}$ are the BRST ghosts (cf., Fig. 1). The superfield $\dot{X}^{\mu}$ charge and transforms as

$$
\stackrel{\circ}{X}^{\mu} \mapsto \stackrel{\circ}{X}^{\mu}+\stackrel{\circ}{\Lambda} \mathfrak{f}_{\beta} \stackrel{\circ}{ }^{\mu} .
$$

We demand our effective actions to be invariant under world volume and target space superdiffeomorphisms.

D. $C P T$ symmetry. The effective field theory is further constrained by the discrete, antilinear $\mathbb{Z}_{2}$ transformation: $C P T$ exchanges $\bar{\theta} \leftrightarrow \theta$ and hence acts as an $R$ parity on the superspace and as usual on the coordinates $\sigma^{a}$. The action can be intuited from using the fact that the $\bar{\theta} \theta$ component of superfields contains the difference operators. Of crucial import is the observation that the gauge sector (5) contains

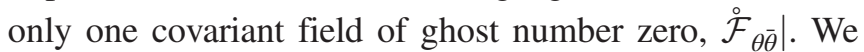
assume that the topological gauge dynamics is consistent with the existence of a vacuum where $\left\langle\dot{\mathcal{F}}_{\theta \bar{\theta}} \mid\right\rangle=-i$, which spontaneously breaks $C P T$. This symmetry-breaking pattern would then explain the emergence of entropy and the associated arrow of time, with $\left\langle\mathcal{\mathcal { F }}_{\theta \bar{\theta}} \mid\right\rangle$ being the order parameter for dissipation.

In fact, much of the discussion below could naively be understood by simply working with a background $U(1)_{\mathrm{T}}$ gauge field $\mathcal{A}_{I}$, restricted to ensure that the field strength component $\left\langle\stackrel{\mathcal{F}}{\theta \bar{\theta}}_{\theta \bar{\theta}} \mid\right\rangle=-i, C P T$ is broken explicitly, and the inflow is biased towards entropy production. We refer to this limit as the MMO limit after Mallick-Moshe-Orland [6]. On physical grounds, since we expect $C P T$ breaking to emerge dynamically rather than being imposed from the beginning, we expect a complete theory to involve the dynamics of $\dot{\mathcal{F}}_{\theta \bar{\theta}}$ as well as saddle points where $\left\langle\dot{\mathcal{F}}_{\theta \bar{\theta}} \mid\right\rangle \neq$ $-i$. Indeed, given the conservation of entropy in superspace, there do not seem to be any fundamental obstructions to gauging the thermal diffeomorphisms and making the superspace gauge fields dynamical.

E. Ghost number conservation. Finally, we impose conservation of the ghost number. In particular, the effective action and physical observables derived from it should have a vanishing net ghost number.

Dissipative hydrodynamic actions and entropy inflow.Having identified the symmetries and the basic fields, we can proceed to construct an effective action, which has to be a topological sigma model. The basic dynamical variables are $\stackrel{\circ}{X}^{\mu}$ (Grassmann coordinates in world volume and target space are aligned, $\Theta=\theta, \bar{\Theta}=\bar{\theta}$ ), but they cannot appear in their bare form, owing to target space diffeomorphism invariance. We pull back the physical target space metric $g_{\mu \nu}$ (with $g_{\Theta \bar{\Theta}}=i, g_{\mu \Theta}=g_{\mu \bar{\Theta}}=0$ ) to a world volume metric

$$
\begin{aligned}
& \stackrel{\circ}{g}_{I J}(z)=g_{\mu \nu}[\stackrel{\circ}{X}(z)] \stackrel{\circ}{\mathcal{D}}_{I} \stackrel{\circ}{X}^{\mu} \stackrel{\circ}{\mathcal{D}}_{J} \stackrel{\circ}{ }^{\nu} \\
& +g_{\Theta \bar{\Theta}}\left(\stackrel{\mathcal{D}}{I}_{I} \stackrel{\mathrm{\Theta}}{\mathcal{D}_{J}} \stackrel{\circ}{\Theta}-\stackrel{\circ}{\mathcal{D}}_{I} \stackrel{\circ}{\Theta} \mathcal{D}_{J} \stackrel{\Theta}{\Theta}\right)
\end{aligned}
$$

and use it to build an effective action. This pullback induces the world volume measure $\sqrt{-\mathrm{g}} / \stackrel{\circ}{\mathbf{z}}$ with $\stackrel{\mathbf{z}}{\text { defined }}$ below in Eq. (10) (see [24]). We also introduce a world 
volume covariant superderivative $\mathfrak{D}_{I}$, which differs from the $U(1)_{T}$ covariant derivative $\stackrel{\mathcal{D}}{I}_{I}$ by terms involving $\stackrel{\circ}{\mathcal{F}}_{I J}$ and $\check{\boldsymbol{\beta}}^{I}$ when acting on world volume tensors, such as to ensure covariance with respect to world volume diffeomorphisms. The precise definition of $\mathfrak{D}_{I}$ will not be material below; all we require is the fact that $\mathfrak{D}_{I}$ allows for integration by parts in superspace [41]. To write an effective action, we begin by writing down the theory capturing the trivial topological sector in terms of the fields introduced so far. We then get physical hydrodynamics by breaking this topological limit by introducing a background source $h_{I J}$ for the energy-momentum tensor $\left(\stackrel{\circ}{g}_{I J} \mapsto \stackrel{\circ}{g}_{I J}+\bar{\theta} \theta h_{I J}\right)$ and $\mathcal{F}_{I}$ for the free energy

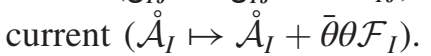

Hydrodynamic transport consistent with the second law is described by effective actions of the form $[24,35]$

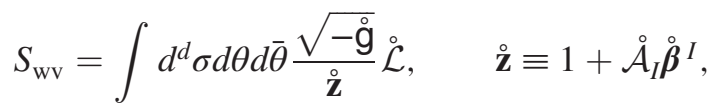

where $\mathcal{L}$ is a scalar functional of the world volume fields, invariant under the following symmetries: (i) ordinary spacetime superdiffeomorphisms, (ii) reparameterizations $z^{I} \mapsto z^{I}+f^{I}\left(\sigma^{a}\right)$ of the world volume supercoordinates, (iii) an antilinear $C P T$ involution, and (iv) ghost number conservation. Invariance under (v) the BRST symmetry and $U(1)_{\mathrm{T}}$ is manifest in the superspace formulation. We define the energy-momentum and free-energy Noether supercurrents:

$$
\begin{aligned}
& \stackrel{\circ}{\mathbf{T}}^{I J} \equiv \frac{2}{\sqrt{-\stackrel{\circ}{\mathrm{g}}}} \frac{\delta}{\delta \stackrel{\mathrm{g}}{I J}_{I J}}(\sqrt{-\stackrel{\mathrm{g}}{\mathcal{L}}}), \\
& \stackrel{\circ}{\mathbf{N}}^{I} \equiv-\frac{\stackrel{\circ}{\mathbf{z}}}{\sqrt{-\stackrel{\circ}{\mathrm{g}}}} \frac{\delta}{\delta \stackrel{\circ}{\mathcal{A}}_{I}}\left(\frac{\sqrt{-\mathrm{g}}}{\stackrel{\circ}{\mathbf{z}}} \stackrel{\circ}{\mathcal{L}}\right) \text {. }
\end{aligned}
$$

In Ref. [35], we show explicitly that the general action of the form (10) consistent with the symmetries mostly reproduces the eightfold classification of ordinary space currents $\left\{\mathbf{T}^{a b}, \mathbf{N}^{a}\right\}$ [42]. In this Letter, we focus on abstract lessons to be learned from the superspace effective action, especially regarding dissipation. Note that these will generically contain fluctuation fields (terms involving $\tilde{X}^{\mu}$ ). The theory thus not only gives classical hydrodynamic constitutive relations but also predicts the form of statistical fluctuations. Supplemental Material [44] provides a simple example illustrating the formal manipulations described in this section.

Physical equations of motion follow from varying the action with respect to the dynamical pullback superfield $\dot{X}^{\mu}$. This enters the action through the metric (9) and therefore leads to energy-momentum conservation in superspace:

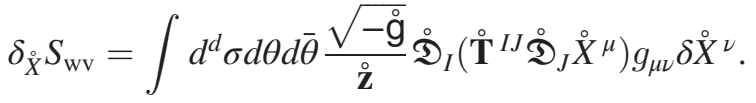

While this equation of motion involves all the supercomponents of the energy-momentum tensor, one can demonstrate equivalence to the classical hydrodynamic equations by noting that it produces correct target space dynamics after projecting down to the bottom component up to fluctuations and ghost bilinears and pushing forward using $X^{\mu}$ :

$$
\begin{aligned}
0 & =\stackrel{\mathfrak{D}}{I}_{I}\left(\stackrel{\circ}{\mathbf{T}}^{I J} \mathfrak{\mathfrak { D }}_{J} \stackrel{\circ}{X}^{\nu}\right) \mid \\
& =\nabla_{\mu} T^{\mu \nu}+\text { ghost bilinears }+ \text { fluctuations. }
\end{aligned}
$$

For the analogue of the adiabaticity equation (1), consider a $U(1)_{T}$ transformation by a gauge parameter $\Lambda$ which transforms the action via

$\delta_{\Lambda} S_{\mathrm{wv}}=\int d^{d} \sigma d \theta d \bar{\theta} \frac{\sqrt{-\mathrm{o}}}{\stackrel{\circ}{\mathbf{z}}} \stackrel{1}{\Lambda}\left\{\frac{1}{2} \stackrel{\circ}{\mathbf{T}}^{I J} £_{\beta} \stackrel{\circ}{\mathrm{g}}_{I J}-\stackrel{\mathfrak{D}}{I}_{I} \stackrel{\circ}{\mathbf{N}}_{I}\right\}$.

We have invoked the $U(1)_{T}$ transformation of the pullback metric (9), inherited from (8), and integrated by parts in superspace. Invariance then implies the superadiabaticity equation:

$$
\mathfrak{D}_{I} \stackrel{\circ}{\mathbf{N}}^{I}-\frac{1}{2} \stackrel{\circ}{\mathbf{T}}^{I J} £_{\beta} \stackrel{\circ}{I J}_{I J}=0 .
$$

This equation is a Bianchi identity for the $U(1)_{\mathrm{T}}$ transformation and, consequently, is insensitive to the gauge dynamics.

The $U(1)_{\mathrm{T}}$ superadiabaticity equation (15) can be decomposed into ordinary and superspace contributions [45]:

$$
\begin{aligned}
& \underbrace{\left(\stackrel{\mathfrak{D}}{a}_{a} \stackrel{\circ}{\mathbf{N}}^{a}-\frac{1}{2} \stackrel{\circ}{\mathbf{T}}^{a b} £_{\beta} \stackrel{\circ}{9}_{a b}\right) \mid}_{\text {classical+fluctuations }}
\end{aligned}
$$

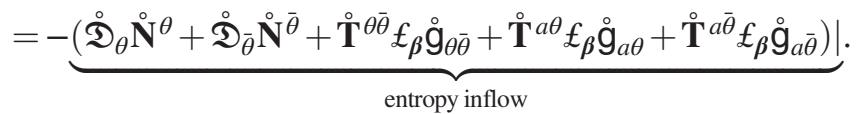

Modulo contributions from the fluctuation fields (and ghost bilinears), the terms we have isolated on the 1.h.s. are precisely the combination appearing on the 1.h.s. of (1) (now in the world volume theory instead of physical spacetime). This naturally suggests interpreting the second set of terms indicated as "entropy inflow" as the part that contributes to the total entropy production $\Delta$. Hence, we identify the r.h.s. of (16) as

$$
\Delta=-\left(\mathfrak{D}_{\theta} \mathbf{N}^{\theta}+\mathfrak{D}_{\bar{\theta}} \mathbf{N}^{\bar{\theta}}\right) \mid+ \text { ghost bilinears },
$$


where we have used the conserved ghost number charge to identify the additional terms as being built out of ghost bilinears (note also that $\mathfrak{f}_{\beta} \stackrel{\circ}{g}_{\theta \bar{\theta}} \mid=0$ ). We interpret this equation as saying that the entropy production is controlled by the Grassmann-odd descendants of the free energy and energy-momentum supertensors [47].

Let us take stock: $U(1)_{\mathrm{T}}$ invariance implies a superBianchi identity which, suitably split up, provides a rationale for entropy production. In particular, the superspace components of the free energy current are primarily responsible for entropy production. This is highly reminiscent of the inflow paradigm for 't Hooft anomalies [7]. The basic idea there is that the effective action for an anomalous symmetry can be presented in a covariant form by viewing the anomalous field theory as the edge or boundary dynamics of a bulk topological field theory. This is familiar from the classic example of quantum Hall edge states (equivalently, the Chern-Simons, WZW correspondence). Our proposition is that entropy production can be viewed in an identical manner in the $U(1)_{\mathrm{T}}$ covariant formalism, with the inflow occurring from Grassmann-odd directions into the physical d.o.f. Despite $\left\{\mathbf{N}^{\theta}, \mathbf{N}^{\bar{\theta}}\right\}$ being ghost currents, their derivatives occurring in (17) are bosonic and physical and capture entropy production.

Our final task is to explain why $\Delta \geq 0$, i.e., the second law. One can, in principle, argue abstractly by invoking the Jarzynski relation $[48,49]$, which follows from the superspace effective action once we assume a $C P T$-breaking pattern $[6,24,50]$. It is more instructive to see this directly by relating to Bhattacharyya's theorem. To this end, we recall that the effective action for dissipative transport is given by [24]

$S_{\mathrm{wv}, \mathrm{diss}}=\int d^{d} \sigma d \theta d \bar{\theta} \frac{\sqrt{-\mathrm{o}}}{\stackrel{\circ}{\mathbf{z}}}\left(-\frac{i}{4}\right) \grave{\boldsymbol{\eta}}^{I J K L} \stackrel{\mathfrak{g}}{I J}_{I J}^{(\bar{\psi})} \stackrel{(\mathfrak{g}}{K L}^{(\psi)}$,

where $\stackrel{\circ}{\mathfrak{g}}_{I J}^{(\bar{\psi})} \equiv \stackrel{\circ}{\mathcal{D}}_{\theta} \stackrel{\circ}{\mathfrak{g}}_{I J}$ and $\stackrel{\circ}{\mathfrak{g}}_{K L}^{(\psi)} \equiv \stackrel{\circ}{\mathcal{D}}_{\bar{\theta}} \stackrel{\circ}{K}_{K L}$. At first sight, these two tensors may appear noncovariant and hence forbidden. However, as is easily verified, the world volume diffeomorphisms specified as symmetry (ii) above are such that these terms are, in fact, covariant and hence allowed (further details may be found in Ref. [35]) [51].

The dissipative tensor $\stackrel{\eta}{\eta}^{I J K L}$ satisfies

$$
\begin{aligned}
& \grave{\boldsymbol{\eta}}^{(I J)(K L)}=(-)^{I J} \grave{\boldsymbol{\eta}}^{(J I)(K L)}=(-)^{K L} \grave{\boldsymbol{\eta}}^{(I J)(L K)} \\
& \stackrel{\circ}{\boldsymbol{\eta}}^{(I J)(K L)}=(-)^{(I+J)(K+L)}\left[\stackrel{\boldsymbol{\eta}}{ }^{(K L)(I J)}\right]^{C P T}
\end{aligned}
$$

but is an otherwise arbitrary covariant functional of the hydrodynamic world volume fields (see [44] for an example). Direct computation of the currents leads to

$$
\Delta=\frac{i}{4}\left(\stackrel{\circ}{\mathcal{F}}_{\theta \bar{\theta}} \mid\right) \boldsymbol{\eta}^{a b c d} £_{\beta} \mathrm{g}_{a b} £_{\beta} \mathbf{g}_{c d}+\text { fluct }+ \text { ghosts. }
$$

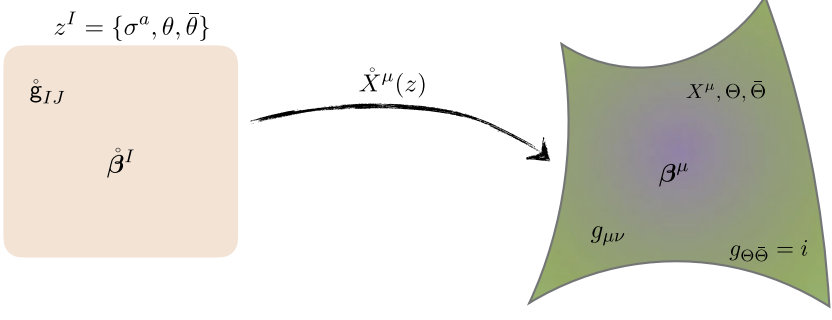

FIG. 1. Illustration of the data for hydrodynamic sigma models. The physical d.o.f. are captured in the target space maps $\stackrel{\circ}{X}^{\mu}\left(\sigma^{a}, \theta, \bar{\theta}\right)$. The world volume geometry is equipped with a rigid supervector field $\stackrel{\circ}{\boldsymbol{\beta}}^{I}$, which pushes forward to the physical thermal vector in spacetime, while the spacetime metric $g_{\mu \nu}, g_{\Theta \bar{\Theta}}=$ $-g_{\bar{\Theta} \Theta}=i$, pulls back to the world volume metric $\stackrel{\circ}{g}_{I J}$. The target space Grassmann coordinates are gauge fixed to be aligned with the world volume ones, $\Theta=\theta, \bar{\Theta}=\bar{\theta}$.

We now invoke the expectation value $\left\langle\stackrel{\circ}{\mathcal{F}}_{\theta \bar{\theta}} \mid\right\rangle=-i$, thus spontaneously breaking $C P T$ as the origin of dissipation [24]. As long as $\boldsymbol{\eta}^{a b c d}$ is a positive-definite map from the space of symmetric two-tensors to symmetric two-tensors, the amount of entropy produced is then non-negative definite, viz., $\Delta \geq 0$, which is precisely the condition established earlier. The easiest way to argue for the positivity of $\boldsymbol{\eta}^{a b c d}$ is by performing the superspace integration in the dissipative effective action which results in an imaginary part of the form $\operatorname{Im}\left(S_{\mathrm{wv}, \text { diss }}\right)=\frac{1}{4} \int d^{d} \sigma \sqrt{-\mathrm{g}} \boldsymbol{\eta}^{a b c d} h_{a b} h_{c d}$. Thus, in order for the work done by the sources to be compatible with a convergent path integral, we need to enforce positivity on $\boldsymbol{\eta}$ at leading order in the derivative expansion (see $[24,27,35])$.

It is a pleasure to thank Shiraz Minwalla, Veronika Hubeny, and Spenta Wadia for discussions on the origins of entropy production in hydrodynamics and beyond. F. M. H. gratefully acknowledges support through a fellowship by the Simons Collaboration "It from Qubit." R. L. gratefully acknowledges support from International Centre for Theoretical Sciences (ICTS), Tata institute of fundamental research, Bengaluru. R. L. also acknowledges his debt to all those who have generously supported and encouraged the pursuit of science in India. M. R. is supported in part by U.S. Department of Energy Grant No. DE-SC0009999 and by the University of California. M. R. thanks TIFR, Mumbai and ICTS, Bengaluru, as well as the organizers of the "It from Qubit workshop" at Bariloche, and "Chaos and Dynamics in Correlated Quantum Matter" at Max Planck Institute for the Physics of Complex Systems, Dresden for hospitality during the course of this work.

Note added.-The work described here was first presented at the "It from Qubit workshop," Bariloche [52]. Recently, we received Ref. [53], which overlaps with the material discussed here. 
*f.m.haehl@gmail.com

†nayagam@gmail.com

‡mukund@physics.ucdavis.edu

[1] L. D. Landau and E. M. Lifshitz, Fluid Mechanics (Pergamon, New York, 1987).

[2] We phrase our discussion for a neutral relativistic fluid. Other conserved charges can be included in a straightforward manner.

[3] V. E. Hubeny, S. Minwalla, and M. Rangamani, arXiv:1107 .5780 .

[4] S. Bhattacharyya, V. E. Hubeny, R. Loganayagam, G. Mandal, S. Minwalla, T. Morita, M. Rangamani, and H. S. Reall, J. High Energy Phys. 06 (2008) 055.

[5] While the arguments presented here apply to near-equilibrium hydrodynamic systems, one might hope that they can be generalized. In particular, the fact that far-fromequilibrium fluctuation relations can be understood in terms of the same symmetries used in our hydrodynamic construction [6] suggests that there might exist a more generally valid framework.

[6] K. Mallick, M. Moshe, and H. Orland, J. Phys. A 44, 095002 (2011).

[7] J. Callan, G. Curtis, and J. A. Harvey, Nucl. Phys. B250, 427 (1985).

[8] N. Banerjee, J. Bhattacharya, S. Bhattacharyya, S. Jain, S. Minwalla, and T. Sharma, J. High Energy Phys. 09 (2012) 046.

[9] K. Jensen, M. Kaminski, P. Kovtun, R. Meyer, A. Ritz, and A. Yarom, Phys. Rev. Lett. 109, 101601 (2012).

[10] F. M. Haehl, R. Loganayagam, and M. Rangamani, Phys. Rev. Lett. 114, 201601 (2015).

[11] V. Iyer and R. M. Wald, Phys. Rev. D 50, 846 (1994).

[12] S. Bhattacharyya, J. High Energy Phys. 07 (2014) 139.

[13] F. M. Haehl, R. Loganayagam, and M. Rangamani, J. High Energy Phys. 05 (2015) 060.

[14] J. S. Schwinger, J. Math. Phys. (N.Y.) 2, 407 (1961).

[15] L. Keldysh, Zh. Eksp. Teor. Fiz. 47, 1515 (1964) [Sov. Phys. JETP 20, 1018 (1965)].

[16] K.-c. Chou, Z.-b. Su, B.-1. Hao, and L. Yu, Phys. Rep. 118, 1 (1985).

[17] R. Kubo, J. Phys. Soc. Jpn. 12, 570 (1957).

[18] P. C. Martin and J. S. Schwinger, Phys. Rev. 115, 1342 (1959).

[19] Understanding the KMS constraints on higher-point functions requires time contours beyond the SK formalism [20].

[20] F. M. Haehl, R. Loganayagam, P. Narayan, A. A. Nizami, and M. Rangamani, J. High Energy Phys. 12 (2017) 154.

[21] P. Kovtun, G. D. Moore, and P. Romatschke, J. High Energy Phys. 07 (2014) 123.

[22] F. M. Haehl, R. Loganayagam, and M. Rangamani, J. High Energy Phys. 01 (2016) 184.

[23] M. Crossley, P. Glorioso, and H. Liu, J. High Energy Phys. 09 (2017) 095.

[24] F. M. Haehl, R. Loganayagam, and M. Rangamani, J. High Energy Phys. 04 (2016) 039.

[25] F. M. Haehl, R. Loganayagam, and M. Rangamani, J. High Energy Phys. 06 (2017) 069.

[26] F. M. Haehl, R. Loganayagam, and M. Rangamani, J. High Energy Phys. 06 (2017) 070.

[27] P. Glorioso and H. Liu, arXiv:1612.07705.
[28] P. Glorioso, M. Crossley, and H. Liu, J. High Energy Phys. 09 (2017) 096.

[29] P. Gao and H. Liu, J. High Energy Phys. 01 (2018) 040.

[30] K. Jensen, N. Pinzani-Fokeeva, and A. Yarom, arXiv:1701 .07436 .

[31] F. M. Haehl, R. Loganayagam, and M. Rangamani, arXiv: 1701.07896

[32] Note that such a structure may be anticipated given the BRST symmetry inherent in effective actions for Langevin systems and stochastic field theories [33,34].

[33] P. Martin, E. Siggia, and H. Rose, Phys. Rev. A 8, 423 (1973).

[34] G. Parisi and N. Sourlas, Nucl. Phys. B206, 321 (1982).

[35] F. M. Haehl, R. Loganayagam, and M. Rangamani, arXiv:1803.11155.

[36] M. Geracie, F. M. Haehl, R. Loganayagam, P. Narayan, D. M. Ramirez, and M. Rangamani, Phys. Rev. D 97, 105023 (2018).

[37] R. Feynman and J. Vernon, Ann. Phys. (N.Y.) 24, 118 (1963).

[38] We will use some of the superdiffeomorphism invariance to simplify the thermal supervector to $\stackrel{\circ}{\boldsymbol{\beta}}^{\theta}=\check{\boldsymbol{\beta}}^{\bar{\theta}}=0=\partial_{\theta} \stackrel{\circ}{\boldsymbol{\beta}}^{a}=$ $\partial_{\bar{\theta}} \stackrel{\circ}{\boldsymbol{\beta}}^{a}$. Note that we consistently use an overcircle to distinguish superfields from ordinary fields.

[39] The high-temperature limit is important so that generators of KMS transformations act locally on the scale of fluid fluctuations and can thus be thought of as generating a local gauge symmetry.

[40] D. Nickel and D. T. Son, New J. Phys. 13, 075010 (2011).

[41] In practice, this requires only that $\mathfrak{D}_{I}$ be compatible with the $U(1)_{\mathrm{T}}$ covariant pullback measure $\mathfrak{D}_{I}(\sqrt{-\mathrm{g}} / \stackrel{\circ}{\mathbf{z}})=0$, which is weaker than being metric compatible as is usually assumed. We demonstrate the existence of such a derivation in Ref. [35].

[42] In the present analysis, we postpone a discussion of both charged and anomaly-induced transport, which require some additional notation and formalism. Corresponding generalizations along the lines of Refs. $[13,43]$ are expected to be straightforward.

[43] F. M. Haehl, R. Loganayagam, and M. Rangamani, J. High Energy Phys. 03 (2014) 034.

[44] See Supplemental Material at http://link.aps.org/ supplemental/10.1103/PhysRevLett.121.051602 for a bulk viscosity example.

[45] Some signs may seem counterintuitive but are a consequence of Grassmann-odd index contractions; we use DeWitt conventions [46].

[46] B. S. DeWitt, Supermanifolds, Cambridge Monographs on Mathematical Physics (Cambridge University Press, Cambridge, United Kingdom, 2012), ISBN 9781139240512 and 9780521423779, http://www.cambridge.org/mw/academic/ subjects/physics/theoretical-physics-and-mathematicalphysics/supermanifolds-2nd-edition?format=AR.

[47] The bosonic part $\Delta_{\text {bos }}=-\left(\mathfrak{D}_{\theta} \mathbf{N}^{\theta}+\mathfrak{D}_{\bar{\theta}} \mathbf{N}^{\bar{\theta}}\right) \mid$ can be obtained directly from the effective action by turning on a suitable source $\mathrm{B}_{\Delta}$; cf. Supplemental Material [44] for an example.

[48] C. Jarzynski, Phys. Rev. Lett. 78, 2690 (1997).

[49] C. Jarzynski, Phys. Rev. E 56, 5018 (1997). 
[50] P. Gaspard, J. Stat. Mech. (2012) P08021.

[51] Note that under reparameterizations $z^{I} \mapsto z^{I}+f^{I}\left(\sigma^{a}\right)$ the world volume connection components $\stackrel{\circ}{\mathcal{C}}_{\theta J}^{I}$ and $\stackrel{\circ}{\mathcal{C}}_{\bar{\theta} J}^{I}$ [and hence $\stackrel{\mathfrak{g}}{I J}_{I J}^{(\bar{\psi})} \equiv \stackrel{\mathfrak{D}}{\theta}_{\theta} \stackrel{\circ}{\mathrm{g}}_{I J}+(-)^{I K} \stackrel{\circ}{\mathcal{C}}_{\theta I}^{K} \stackrel{\circ}{\mathrm{g}}_{K J}+(-)^{K(I+J)+I J} \stackrel{\circ}{\mathcal{C}}_{\theta J}^{K} \stackrel{\circ}{\mathrm{g}}_{I K}$, etc.] are covariant.
[52] M. Rangamani, http://fisica.cab.cnea.gov.ar/itfromqubit/ images/talks/rangamani.compressed.pdf, https://www.youtube .com/watch?v=W9wkE72vGlA.

[53] K. Jensen, R. Marjieh, N. Pinzani-Fokeeva, and A. Yarom, arXiv:1803.07070. 